\title{
Syntheses and Structures of a Macrocyclic $\beta$ - Diketimine and Its Zinc and Copper Complexes
}

\author{
Su Yeon Lee ${ }^{a}$, Sung Jae Na ${ }^{a}$, Heon Yong Kwon ${ }^{a}$, Bun Yeoul Lee ${ }^{a}$, and Sang Ook \\ Kang $^{b}$
}

${ }^{a}$ Department of Molecular Science and Technology, Ajou University, Suwon 443-749

Korea and ${ }^{b}$ Department of Chemistry, Korea University, 208 Seochang, Chochiwon, Chung-nam, 339-700, Korea

bunyeoul@ajou.ac.kr

Supporting Information 


\section{Experimentals}

All manipulations were performed under an inert atmosphere using standard glove box and Schlenk techniques. Pentane and $\mathrm{C}_{6} \mathrm{D}_{6}$ were distilled from benzophenone ketyl. Ethanol was dried by literature method by using sodium and diethyl phthalate (Armarego, W. L. F.; Perrin, D. D.; Purification of Laboratory Chemicals $4^{\text {th }}$ Ed, Butterworth-Heinemann: Singapore, 1996; p 209). Pyridine was dried over $\mathrm{CaH}_{2} .{ }^{1} \mathrm{H}$ NMR (400 MHz) and ${ }^{13} \mathrm{C}$ NMR (100 MHz) spectra were recorded on a Varian Mercury plus 400. Elemental analyses were carried out on a Perkin-Elmer $2400 \mathrm{CHN}$ microanalyzer.

Compound 4. To a solution of 4,4"-diamino-o-terphenyl $(2.00 \mathrm{~g}, 7.68 \mathrm{mmol})$ in benzene $(100 \mathrm{~mL})$ was added 2,4-pentanedione $(4.62 \mathrm{~g}, 46.0 \mathrm{mmol})$ and $p$ toluenesulfonic acid (73 mg, $0.38 \mathrm{mmol}, 5 \mathrm{~mol} \%)$. The solution was refluxed with Dean-Stock apparatus for 1 day. The resulting yellow solution was washed with saturated aqueous $\mathrm{NaHCO}_{3}$ solution and the solvent was removed with rotary evaporator. The residue was triturated with hexane to give yellow solid (3.0 g, $92 \%)$. M.p. $152{ }^{\circ} \mathrm{C} .{ }^{1} \mathrm{H}-\mathrm{NMR}\left(\mathrm{CDCl}_{3}\right): \delta 12.34$ (s, $\left.2 \mathrm{H}, \mathrm{NH}\right), 7.35$ (s, $4 \mathrm{H}$, internal-benzene-H), $7.02(\mathrm{~d}, J=7.6 \mathrm{~Hz}, 4 \mathrm{H}$, peripheral-benzene-H), 6.89 (d, $J=9.2 \mathrm{~Hz}, 4 \mathrm{H}$, peripheralbenzene-H), $5.13(\mathrm{~s}, 2 \mathrm{H}, \mathrm{CH}), 2.07\left(\mathrm{~s}, 6 \mathrm{H}, \mathrm{CH}_{3}\right), 1.96\left(\mathrm{~s}, 6 \mathrm{H}, \mathrm{CH}_{3}\right) \mathrm{ppm} .{ }^{13} \mathrm{C}\left\{{ }^{1} \mathrm{H}\right\}$ $\operatorname{NMR}\left(\mathrm{C}_{6} \mathrm{D}_{6}\right): \delta 195.86(\mathrm{CO}), 159.70,139.43,138.32,137.15,130.36,130.32,127.57$, 123.83, 97.77, $29.30\left(\mathrm{CH}_{3}\right), 20.07\left(\mathrm{CH}_{3}\right)$ ppm. Anal. Calc. $\left(\mathrm{C}_{28} \mathrm{H}_{28} \mathrm{~N}_{2} \mathrm{O}_{2}\right): \mathrm{C}, 79.2 ; \mathrm{H}$, $6.65 ; \mathrm{N}, 6.60 \%$. Found: C, 79.3; H, 6.38; N, $6.42 \%$.

HCl salt of 3. Concentrated $\mathrm{HCl}(2.0 \mathrm{~mL}, 23 \mathrm{mmol})$ was added to 4,4"-diamino-oterphenyl $(1.50 \mathrm{~g}, 5.76 \mathrm{mmol})$ in ethanol $(10 \mathrm{~mL})$. The mixture was stirred for 30 minutes to give a white solid which is isolated by filtration and washing with diethyl 
ether $(1.64 \mathrm{~g}, 85 \%)$.

Compound 5. Equimolar amount of the $\mathrm{HCl}$ salt of 4,4"-diamino-o-terphenyl (1.38 g, $3.46 \mathrm{mmol})$ and $4(1.47 \mathrm{~g}, 3.46 \mathrm{mmol})$ was dissolved in anhydrous ethanol $(450 \mathrm{~mL})$. The solution was stirred at $70 \square$ for 5 days to give a yellow precipitates. After the solution was cooled to room temperature, the solid was collected by filtration. The solid was dispersed in ethanol $(30 \mathrm{~mL})$ and saturated aqueous $\mathrm{NaHCO}_{3}$ solution $(20 \mathrm{~mL})$ was added. The mixture was stirred for 2 minutes. The compound was extracted with benzene $(100 \mathrm{~mL} \times 2)$. Solvent was removed to give yellow crystalline solid $(1.02 \mathrm{~g}$, $42 \%$ ) which is pure by the analysis of NMR spectra. Single crystals are obtained by vapor phase addition of pentane to a benzene solution. M.p. decomposed $>300{ }^{\circ} \mathrm{C} .{ }^{1} \mathrm{H}$ $\operatorname{NMR}\left(\mathrm{C}_{6} \mathrm{D}_{6}\right): \delta 12.95(\mathrm{~s}, 2 \mathrm{H}, \mathrm{NH}), 7.41(\mathrm{dd}, J=5.6,3.2 \mathrm{~Hz}, 4 \mathrm{H}$, internal-benzene-H), 7.21 (dd, $J=5.6,3.2 \mathrm{~Hz}, 4 \mathrm{H}$, internal-benzene-H), $7.14(\mathrm{~d}, J=8.4 \mathrm{~Hz}, 8 \mathrm{H}$, peripheralbenzene-H), 6.58 (d, $J=8.4 \mathrm{~Hz}, 8 \mathrm{H}$, peripheral-benzene-H), 4.75 (s, $2 \mathrm{H}, \mathrm{CH}), 1.81$ (s, $\left.12 \mathrm{H}, \mathrm{CH}_{3}\right)$ ppm. ${ }^{13} \mathrm{C}\left\{{ }^{1} \mathrm{H}\right\}$ NMR $\left(\mathrm{C}_{6} \mathrm{D}_{6}\right): \delta 158.89,144.59,140.65,137.08,131.09$, 129.38, 122.81, $98.17(\mathrm{CH}), 20.74\left(\mathrm{CH}_{3}\right)$ ppm. Anal. Calc. $\left(\mathrm{C}_{46} \mathrm{H}_{40} \mathrm{~N}_{4}\right): \mathrm{C}, 85.2 ; \mathrm{H}, 6.21$; N, $8.63 \%$. Found: C, 84.9; H, 6.60; N, $8.26 \%$.

Compound 6. A solution of $\mathrm{ZnEt}_{2}(126 \mathrm{mg}, 0.173 \mathrm{mmol})$ in pentane was layered to a solution of 5 (50 mg, $0.069 \mathrm{mmol})$ in benzene and pyridine (v/v, 10:1). Pale yellow crystals were deposited after overnight (39 mg, 57\%). Anal. Calc. $\left(\mathrm{C}_{60} \mathrm{H}_{58} \mathrm{~N}_{6} \mathrm{Zn}_{2}\right): \mathrm{C}$, $72.5 ; \mathrm{H}, 5.89 ; \mathrm{N}, 8.46 \%$. Found: C, 72.4; H, 5.58; N, $8.12 \%$.

Compound 7. A solution of $\mathrm{Cu}(\mathrm{I}) \mathrm{O}^{t} \mathrm{Bu}(19 \mathrm{mg}, 0.14 \mathrm{mmol})$ in benzene was layered to a solution of 5 (50 mg,0.069 mmol) in benzene. Pale yellow crystals were deposited after 2 days $(47 \mathrm{mg}, 62 \%)$. Anal. Calc. $\left(\mathrm{C}_{58} \mathrm{H}_{50} \mathrm{Cu}_{2} \mathrm{~N}_{4} \cdot 2 \mathrm{C}_{6} \mathrm{H}_{6}\right): \mathrm{C}, 77.4 ; \mathrm{H}, 5.76 ; \mathrm{N}, 5.16 \%$. Found: C, 77.0; H, 5.41; N, $5.01 \%$. 


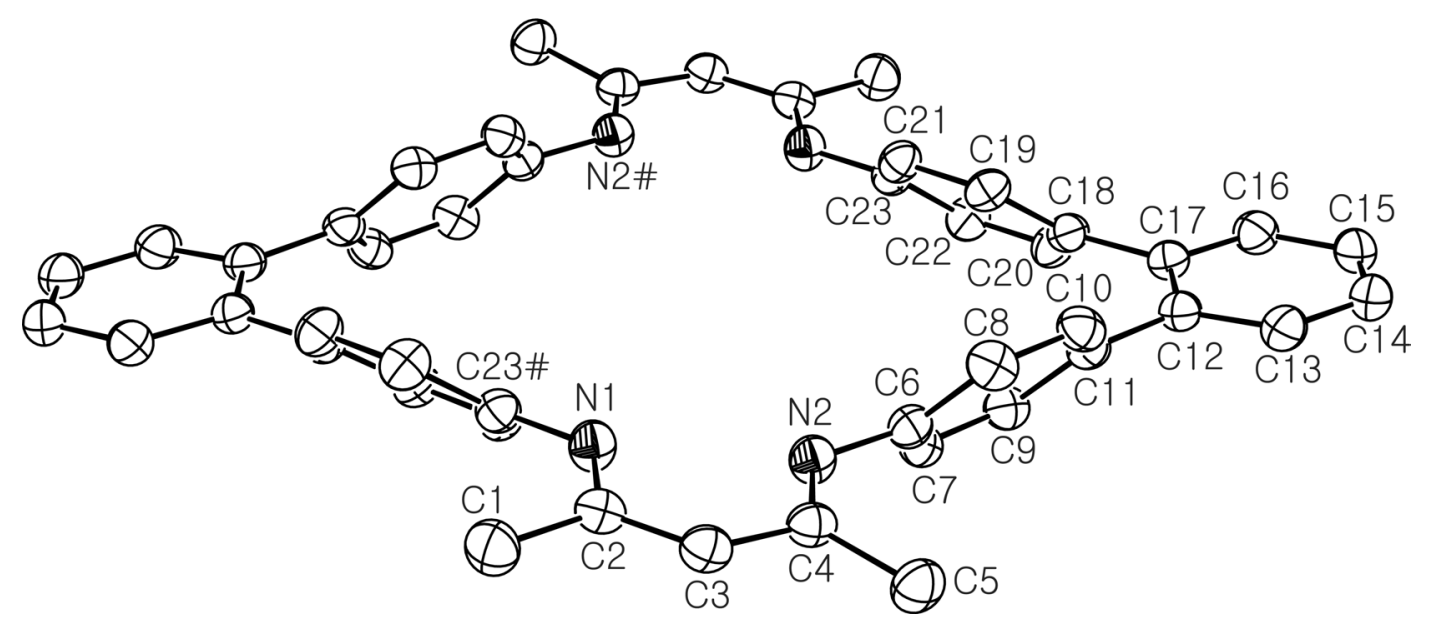

Table 1. Atomic coordinates (x 10^4) and equivalent isotropic displacement parameters $\left(\mathrm{A}^{\wedge} 2 \times 10^{\wedge} 3\right) . \mathrm{U}(\mathrm{eq})$ is defined as one third of the trace of the orthogonalized Uij tensor.

\begin{tabular}{lcccc}
\hline & $\mathrm{x}$ & $\mathrm{y}$ & $\mathrm{z}$ & $\mathrm{U}(\mathrm{eq})$ \\
\hline $\mathrm{N}(1)$ & $6775(3)$ & $8781(2)$ & $4662(1)$ & $58(1)$ \\
$\mathrm{N}(2)$ & $7285(3)$ & $8730(2)$ & $6457(1)$ & $55(1)$ \\
$\mathrm{C}(1)$ & $7641(4)$ & $10902(2)$ & $4250(2)$ & $73(1)$ \\
$\mathrm{C}(2)$ & $7473(3)$ & $10072(2)$ & $4950(2)$ & $54(1)$ \\
$\mathrm{C}(5)$ & $8358(4)$ & $10821(2)$ & $7589(2)$ & $69(1)$ \\
$\mathrm{C}(3)$ & $7989(3)$ & $10626(2)$ & $5880(2)$ & $55(1)$ \\
$\mathrm{C}(4)$ & $7867(3)$ & $9978(2)$ & $6626(2)$ & $52(1)$ \\
$\mathrm{C}(23)$ & $4008(3)$ & $1966(2)$ & $6250(2)$ & $52(1)$ \\
$\mathrm{C}(21)$ & $3187(3)$ & $1792(2)$ & $6977(2)$ & $60(1)$ \\
$\mathrm{C}(19)$ & $4017(3)$ & $2569(2)$ & $7848(2)$ & $58(1)$ \\
$\mathrm{C}(18)$ & $5685(3)$ & $3560(2)$ & $8037(2)$ & $50(1)$ \\
$\mathrm{C}(20)$ & $6469(3)$ & $3745(2)$ & $7293(2)$ & $53(1)$ \\
$\mathrm{C}(22)$ & $5658(3)$ & $2969(2)$ & $6417(2)$ & $53(1)$ \\
$\mathrm{C}(17)$ & $6594(3)$ & $4343(2)$ & $9002(1)$ & $50(1)$ \\
$\mathrm{C}(16)$ & $6679(3)$ & $3698(2)$ & $9731(2)$ & $60(1)$ \\
$\mathrm{C}(15)$ & $7578(3)$ & $4343(3)$ & $10646(2)$ & $66(1)$ \\
$\mathrm{C}(14)$ & $8419(4)$ & $5639(3)$ & $10840(2)$ & $68(1)$ \\
$\mathrm{C}(13)$ & $8329(3)$ & $6291(2)$ & $10133(2)$ & $63(1)$ \\
& & & &
\end{tabular}




$\begin{array}{lllll}\mathrm{C}(12) & 7413(3) & 5682(2) & 9209(1) & 51(1) \\ \mathrm{C}(11) & 7355(3) & 6472(2) & 8495(1) & 50(1) \\ \mathrm{C}(9) & 8980(3) & 7369(2) & 8463(2) & 57(1) \\ \mathrm{C}(7) & 8963(3) & 8119(2) & 7812(2) & 59(1) \\ \mathrm{C}(6) & 7300(3) & 8029(2) & 7169(2) & 51(1) \\ \mathrm{C}(8) & 5664(3) & 7139(2) & 7187(2) & 53(1) \\ \mathrm{C}(10) & 5686(3) & 6382(2) & 7836(2) & 54(1) \\ \mathrm{C}(31) & 4071(4) & 621(3) & 514(2) & 85(1) \\ \mathrm{C}(32) & 3353(4) & 185(3) & -425(2) & 89(1) \\ \mathrm{C}(33) & 5720(5) & 431(3) & 931(2) & 92(1) \\ \mathrm{C}(41) & 1030(30) & 4222(18) & 4740(20) & 155(10) \\ \mathrm{C}\left(41^{\prime}\right) & 820(30) & 4503(17) & 4382(16) & 113(5) \\ \mathrm{C}(42) & -322(19) & 4866(13) & 4072(5) & 167(3) \\ \mathrm{C}(43) & 1235(10) & 4514(9) & 5450(14) & 177(4) \\ & & & & \end{array}$

Table 2. Bond lengths [A] and angles [deg].

\begin{tabular}{llll}
\cline { 2 - 3 } $\mathrm{N}(1)-\mathrm{C}(2)$ & $1.354(3)$ & $\mathrm{N}(1)-\mathrm{C}(23) \# 1$ & $1.407(3)$ \\
$\mathrm{N}(2)-\mathrm{C}(4)$ & $1.302(3)$ & $\mathrm{N}(2)-\mathrm{C}(6)$ & $1.413(3)$ \\
$\mathrm{C}(1)-\mathrm{C}(2)$ & $1.501(3)$ & $\mathrm{C}(2)-\mathrm{C}(3)$ & $1.370(3)$ \\
$\mathrm{C}(5)-\mathrm{C}(4)$ & $1.514(3)$ & $\mathrm{C}(3)-\mathrm{C}(4)$ & $1.425(3)$ \\
$\mathrm{C}(23)-\mathrm{C}(21)$ & $1.381(3)$ & $\mathrm{C}(23)-\mathrm{C}(22)$ & $1.391(3)$ \\
$\mathrm{C}(23)-\mathrm{N}(1) \# 1$ & $1.407(3)$ & $\mathrm{C}(21)-\mathrm{C}(19)$ & $1.376(3)$ \\
$\mathrm{C}(19)-\mathrm{C}(18)$ & $1.390(3)$ & $\mathrm{C}(18)-\mathrm{C}(20)$ & $1.390(3)$ \\
$\mathrm{C}(18)-\mathrm{C}(17)$ & $1.493(3)$ & $\mathrm{C}(20)-\mathrm{C}(22)$ & $1.380(3)$ \\
$\mathrm{C}(17)-\mathrm{C}(16)$ & $1.393(3)$ & $\mathrm{C}(17)-\mathrm{C}(12)$ & $1.407(3)$ \\
$\mathrm{C}(16)-\mathrm{C}(15)$ & $1.389(3)$ & $\mathrm{C}(15)-\mathrm{C}(14)$ & $1.368(3)$ \\
$\mathrm{C}(14)-\mathrm{C}(13)$ & $1.370(3)$ & $\mathrm{C}(13)-\mathrm{C}(12)$ & $1.394(3)$ \\
$\mathrm{C}(12)-\mathrm{C}(11)$ & $1.482(3)$ & $\mathrm{C}(11)-\mathrm{C}(9)$ & $1.386(3)$ \\
$\mathrm{C}(11)-\mathrm{C}(10)$ & $1.403(3)$ & $\mathrm{C}(9)-\mathrm{C}(7)$ & $1.374(3)$ \\
$\mathrm{C}(7)-\mathrm{C}(6)$ & $1.388(3)$ & $\mathrm{C}(6)-\mathrm{C}(8)$ & $1.382(3)$ \\
$\mathrm{C}(8)-\mathrm{C}(10)$ & $1.377(3)$ & $\mathrm{C}(31)-\mathrm{C}(33)$ & $1.355(4)$ \\
$\mathrm{C}(31)-\mathrm{C}(32)$ & $1.365(4)$ & $\mathrm{C}(32)-\mathrm{C}(33) \# 2$ & $1.354(4)$ \\
$\mathrm{C}(33)-\mathrm{C}(32) \# 2$ & $1.354(4)$ & $\mathrm{C}(41)-\mathrm{C}(43)$ & $1.04(2)$ \\
$\mathrm{C}(41)-\mathrm{C}(42)$ & $1.65(2)$ & $\mathrm{C}(41)-\mathrm{C}(42)$ & $1.088(17)$
\end{tabular}




\begin{tabular}{|c|c|c|c|}
\hline$C\left(41^{\prime}\right)-C(43)$ & $1.57(2)$ & $\mathrm{C}(42)-\mathrm{C}(43) \# 3$ & $1.324(9)$ \\
\hline$C(43)-C(42) \# 3$ & $1.324(9)$ & $\mathrm{C}(2)-\mathrm{N}(1)-\mathrm{C}(23) \# 1$ & $129.2(2)$ \\
\hline $\mathrm{C}(4)-\mathrm{N}(2)-\mathrm{C}(6)$ & $122.83(18)$ & $\mathrm{N}(1)-\mathrm{C}(2)-\mathrm{C}(3)$ & $120.1(2)$ \\
\hline $\mathrm{N}(1)-\mathrm{C}(2)-\mathrm{C}(1)$ & $120.1(2)$ & $\mathrm{C}(3)-\mathrm{C}(2)-\mathrm{C}(1)$ & $119.8(2)$ \\
\hline$C(2)-C(3)-C(4)$ & $126.7(2)$ & $\mathrm{N}(2)-\mathrm{C}(4)-\mathrm{C}(3)$ & $120.2(2)$ \\
\hline $\mathrm{N}(2)-\mathrm{C}(4)-\mathrm{C}(5)$ & $123.5(2)$ & $\mathrm{C}(3)-\mathrm{C}(4)-\mathrm{C}(5)$ & $116.3(2)$ \\
\hline$C(21)-C(23)-C(22)$ & $118.3(2)$ & $\mathrm{C}(21)-\mathrm{C}(23)-\mathrm{N}(1) \# 1$ & $123.6(2)$ \\
\hline $\mathrm{C}(22)-\mathrm{C}(23)-\mathrm{N}(1) \# 1$ & $118.1(2)$ & $C(19)-C(21)-C(23)$ & $120.6(2)$ \\
\hline $\mathrm{C}(21)-\mathrm{C}(19)-\mathrm{C}(18)$ & $122.3(2)$ & $\mathrm{C}(20)-\mathrm{C}(18)-\mathrm{C}(19)$ & $116.5(2)$ \\
\hline $\mathrm{C}(20)-\mathrm{C}(18)-\mathrm{C}(17)$ & 122.70(19) & $\mathrm{C}(19)-\mathrm{C}(18)-\mathrm{C}(17)$ & $120.7(2)$ \\
\hline$C(22)-C(20)-C(18)$ & $121.8(2)$ & $\mathrm{C}(20)-\mathrm{C}(22)-\mathrm{C}(23)$ & $120.5(2)$ \\
\hline$C(16)-C(17)-C(12)$ & 118.7(2) & $\mathrm{C}(16)-\mathrm{C}(17)-\mathrm{C}(18)$ & $117.7(2)$ \\
\hline $\mathrm{C}(12)-\mathrm{C}(17)-\mathrm{C}(18)$ & 123.63(19) & $C(15)-C(16)-C(17)$ & $121.5(2)$ \\
\hline $\mathrm{C}(14)-\mathrm{C}(15)-\mathrm{C}(16)$ & $119.5(2)$ & $\mathrm{C}(15)-\mathrm{C}(14)-\mathrm{C}(13)$ & $119.7(2)$ \\
\hline $\mathrm{C}(14)-\mathrm{C}(13)-\mathrm{C}(12)$ & $122.5(2)$ & $\mathrm{C}(13)-\mathrm{C}(12)-\mathrm{C}(17)$ & $118.0(2)$ \\
\hline $\mathrm{C}(13)-\mathrm{C}(12)-\mathrm{C}(11)$ & $118.6(2)$ & $\mathrm{C}(17)-\mathrm{C}(12)-\mathrm{C}(11)$ & $123.39(18)$ \\
\hline C(9)-C(11)-C(10) & $116.5(2)$ & $\mathrm{C}(9)-\mathrm{C}(11)-\mathrm{C}(12)$ & 120.61(19) \\
\hline $\mathrm{C}(10)-\mathrm{C}(11)-\mathrm{C}(12)$ & $122.88(19)$ & $\mathrm{C}(7)-\mathrm{C}(9)-\mathrm{C}(11)$ & $121.8(2)$ \\
\hline $\mathrm{C}(9)-\mathrm{C}(7)-\mathrm{C}(6)$ & $121.2(2)$ & $\mathrm{C}(8)-\mathrm{C}(6)-\mathrm{C}(7)$ & $118.0(2)$ \\
\hline $\mathrm{C}(8)-\mathrm{C}(6)-\mathrm{N}(2)$ & $120.08(19)$ & $\mathrm{C}(7)-\mathrm{C}(6)-\mathrm{N}(2)$ & $121.7(2)$ \\
\hline$C(10)-C(8)-C(6)$ & $120.7(2)$ & $\mathrm{C}(8)-\mathrm{C}(10)-\mathrm{C}(11)$ & $121.8(2)$ \\
\hline $\mathrm{C}(33)-\mathrm{C}(31)-\mathrm{C}(32)$ & 119.1(3) & $\mathrm{C}(33) \# 2-\mathrm{C}(32)-\mathrm{C}(31)$ & $120.3(3)$ \\
\hline C(32)\#2-C(33)-C(31) & $120.6(3)$ & $C(43)-C(41)-C(42)$ & $116.0(17)$ \\
\hline $\mathrm{C}(42)-\mathrm{C}\left(41^{\prime}\right)-\mathrm{C}(43)$ & $118.6(15)$ & $\mathrm{C}\left(41^{\prime}\right)-\mathrm{C}(42)-\mathrm{C}(43) \# 3$ & $124.6(13)$ \\
\hline $\mathrm{C}\left(41^{\prime}\right)-\mathrm{C}(42)-\mathrm{C}(41)$ & $16.0(13)$ & $\mathrm{C}(43) \# 3-\mathrm{C}(42)-\mathrm{C}(41)$ & $111.7(10)$ \\
\hline $\mathrm{C}(41)-\mathrm{C}(43)-\mathrm{C}(42) \# 3$ & 131.0(16) & $\mathrm{C}(41)-\mathrm{C}(43)-\mathrm{C}\left(41^{\prime}\right)$ & $18.4(16)$ \\
\hline $\mathrm{C}(42) \# 3-\mathrm{C}(43)-\mathrm{C}\left(41^{\prime}\right)$ & 116.1(9) & & \\
\hline
\end{tabular}

Symmetry transformations used to generate equivalent atoms:

Table 4. Anisotropic displacement parameters $\left(\mathrm{A}^{\wedge} 2 \times 10^{\wedge} 3\right)$. The anisotropic displacement factor exponent takes the form: $-2 \mathrm{pi}^{\wedge} 2\left[\mathrm{~h}^{\wedge} 2 \mathrm{a}^{* \wedge} 2 \mathrm{U} 11+\ldots+2 \mathrm{~h} \mathrm{k} \mathrm{a}^{*} \mathrm{~b}^{*}\right.$ U12 ]

$\mathrm{U} 11$

$\mathrm{U} 22$

$\mathrm{U} 33$

$\mathrm{U} 23$

$\mathrm{U} 13$

$\mathrm{U} 12$ 


\begin{tabular}{|c|c|c|c|c|c|c|}
\hline $\mathrm{N}(1)$ & $7(1)$ & $48(1)$ & $53(1)$ & $8(1)$ & 11(1) & 11(1) \\
\hline $\mathrm{N}(2)$ & $61(1)$ & $46(1)$ & $53(1)$ & $7(1)$ & $10(1)$ & 11(1) \\
\hline $\mathrm{C}(1)$ & $92(2)$ & $56(2)$ & $73(2)$ & $20(1)$ & $22(1)$ & $22(1)$ \\
\hline$C(2)$ & $50(1)$ & $49(1)$ & $62(2)$ & 11(1) & $12(1)$ & $17(1)$ \\
\hline$C(5)$ & $82(2)$ & $55(1)$ & $64(2)$ & $3(1)$ & $19(1)$ & $17(1)$ \\
\hline$C(3)$ & $56(1)$ & $42(1)$ & $63(2)$ & $6(1)$ & $11(1)$ & $11(1)$ \\
\hline$C(4)$ & $48(1)$ & $45(1)$ & $58(1)$ & $4(1)$ & $11(1)$ & $13(1)$ \\
\hline $\mathrm{C}(23)$ & $55(1)$ & $49(1)$ & $51(1)$ & $7(1)$ & $11(1)$ & $17(1)$ \\
\hline$C(21)$ & $50(1)$ & $62(2)$ & $61(2)$ & $6(1)$ & $14(1)$ & $7(1)$ \\
\hline $\mathrm{C}(19)$ & $56(1)$ & $62(2)$ & $57(1)$ & $11(1)$ & $23(1)$ & $13(1)$ \\
\hline $\mathrm{C}(18)$ & $53(1)$ & $50(1)$ & $52(1)$ & $10(1)$ & $18(1)$ & $19(1)$ \\
\hline$C(20)$ & $56(1)$ & $44(1)$ & $58(1)$ & $7(1)$ & $20(1)$ & 11(1) \\
\hline $\mathrm{C}(22)$ & $63(1)$ & $45(1)$ & $53(1)$ & $10(1)$ & $21(1)$ & $15(1)$ \\
\hline$C(17)$ & $48(1)$ & $54(1)$ & $49(1)$ & $10(1)$ & $15(1)$ & $18(1)$ \\
\hline$C(16)$ & $62(1)$ & $65(2)$ & $61(2)$ & $19(1)$ & $20(1)$ & $23(1)$ \\
\hline$C(15)$ & $71(2)$ & $88(2)$ & $52(2)$ & $23(1)$ & $20(1)$ & $38(1)$ \\
\hline $\mathrm{C}(14)$ & $70(2)$ & $86(2)$ & $48(1)$ & $5(1)$ & $8(1)$ & $35(1)$ \\
\hline$C(13)$ & $65(2)$ & $64(2)$ & $56(2)$ & $2(1)$ & $10(1)$ & $23(1)$ \\
\hline $\mathrm{C}(12)$ & $49(1)$ & $57(1)$ & $49(1)$ & $7(1)$ & 11(1) & $21(1)$ \\
\hline $\mathrm{C}(11)$ & $54(1)$ & $45(1)$ & $50(1)$ & $4(1)$ & $13(1)$ & $18(1)$ \\
\hline $\mathrm{C}(9)$ & $51(1)$ & $53(1)$ & $61(1)$ & $8(1)$ & $5(1)$ & $16(1)$ \\
\hline$C(7)$ & $52(1)$ & $52(1)$ & $67(2)$ & $10(1)$ & $9(1)$ & $8(1)$ \\
\hline$C(6)$ & $57(1)$ & $45(1)$ & $48(1)$ & $3(1)$ & $12(1)$ & $15(1)$ \\
\hline$C(8)$ & $51(1)$ & $50(1)$ & $53(1)$ & $5(1)$ & $5(1)$ & $14(1)$ \\
\hline $\mathrm{C}(10)$ & $47(1)$ & $52(1)$ & $58(1)$ & $6(1)$ & $10(1)$ & $12(1)$ \\
\hline $\mathrm{C}(31)$ & $87(2)$ & $76(2)$ & $88(2)$ & $-3(2)$ & $32(2)$ & $23(2)$ \\
\hline$C(32)$ & $80(2)$ & $88(2)$ & $91(2)$ & $8(2)$ & $7(2)$ & $27(2)$ \\
\hline$C(33)$ & $98(2)$ & $104(2)$ & $60(2)$ & $-2(2)$ & $10(2)$ & $27(2)$ \\
\hline $\mathrm{C}(41)$ & $198(17)$ & $71(8)$ & $180(20)$ & $-18(12)$ & 73(17) & $14(8)$ \\
\hline$C\left(41^{\prime}\right)$ & $83(7)$ & $109(10)$ & $148(15)$ & $21(8)$ & $47(8)$ & $18(6)$ \\
\hline $\mathrm{C}(42)$ & $126(6)$ & $176(7)$ & $155(5)$ & $38(6)$ & $31(6)$ & $-29(5)$ \\
\hline$C(43)$ & $103(4)$ & $126(6)$ & 281(14) & $38(6)$ & $20(8)$ & $27(4)$ \\
\hline
\end{tabular}

Table 4. Hydrogen coordinates $\left(\mathrm{x} 10^{\wedge} 4\right)$ and isotropic displacement parameters $\left(\mathrm{A}^{\wedge} 2\right.$ x $\left.10^{\wedge} 3\right)$. 


\begin{tabular}{|c|c|c|c|c|}
\hline & $\mathrm{x}$ & $\mathrm{y}$ & $\mathrm{z}$ & $\mathrm{U}(\mathrm{eq})$ \\
\hline HN1 & $6540(40)$ & $8380(30)$ & $5139(19)$ & $87(9)$ \\
\hline $\mathrm{H}(1 \mathrm{~A})$ & 7236 & 10370 & 3635 & 109 \\
\hline $\mathrm{H}(1 \mathrm{~B})$ & 8934 & 11405 & 4367 & 109 \\
\hline $\mathrm{H}(1 \mathrm{C})$ & 6859 & 11462 & 4301 & 109 \\
\hline $\mathrm{H}(5 \mathrm{~A})$ & 8242 & 10291 & 8037 & 103 \\
\hline $\mathrm{H}(5 \mathrm{~B})$ & 7511 & 11338 & 7605 & 103 \\
\hline $\mathrm{H}(5 \mathrm{C})$ & 9634 & 11366 & 7736 & 103 \\
\hline $\mathrm{H}(3)$ & 8469 & 11515 & 6042 & 66 \\
\hline $\mathrm{H}(21)$ & 2063 & 1144 & 6876 & 72 \\
\hline $\mathrm{H}(19)$ & 3442 & 2426 & 8328 & 70 \\
\hline $\mathrm{H}(20)$ & 7572 & 4410 & 7389 & 63 \\
\hline $\mathrm{H}(22)$ & 6217 & 3118 & 5933 & 64 \\
\hline $\mathrm{H}(16)$ & 6122 & 2816 & 9601 & 72 \\
\hline $\mathrm{H}(15)$ & 7608 & 3896 & 11123 & 79 \\
\hline $\mathrm{H}(14)$ & 9048 & 6075 & 11448 & 81 \\
\hline $\mathrm{H}(13)$ & 8900 & 7172 & 10275 & 76 \\
\hline $\mathrm{H}(9)$ & 10113 & 7466 & 8894 & 68 \\
\hline $\mathrm{H}(7)$ & 10086 & 8697 & 7802 & 71 \\
\hline $\mathrm{H}(8)$ & 4535 & 7050 & 6757 & 64 \\
\hline $\mathrm{H}(10)$ & 4564 & 5795 & 7837 & 65 \\
\hline $\mathrm{H}(31)$ & 3436 & 1044 & 862 & 102 \\
\hline $\mathrm{H}(32)$ & 2223 & 311 & -719 & 107 \\
\hline $\mathrm{H}(33)$ & 6220 & 725 & 1570 & 110 \\
\hline $\mathrm{H}(41)$ & 1588 & 3631 & 4491 & 186 \\
\hline $\mathrm{H}\left(41^{\prime}\right)$ & 1538 & 4194 & 4020 & 136 \\
\hline $\mathrm{H}(42)$ & -433 & 4803 & 3438 & 200 \\
\hline $\mathrm{H}(43)$ & 2224 & 4306 & 5814 & 212 \\
\hline
\end{tabular}

X-Ray Report for 6 


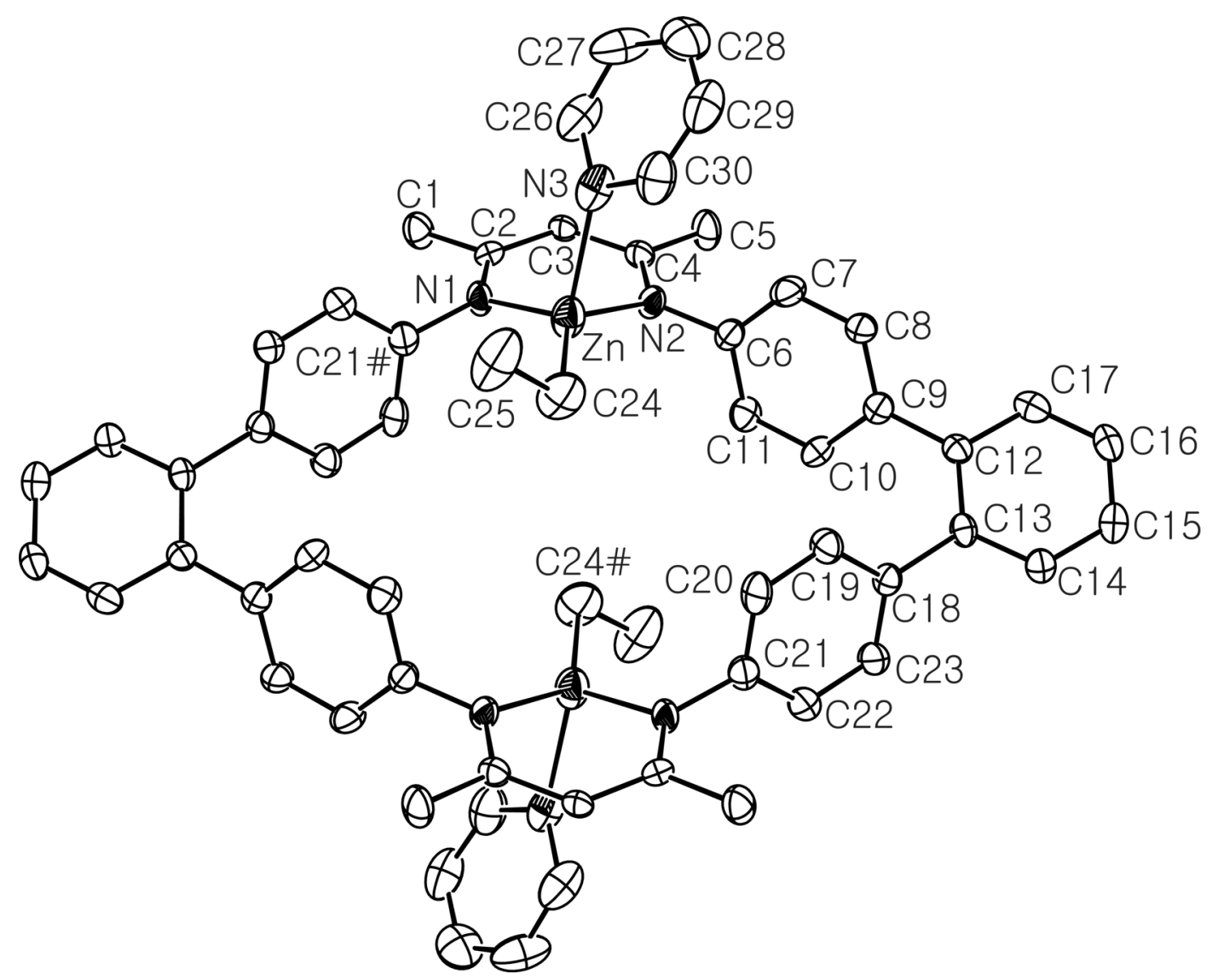

Table 1. Atomic coordinates ( $\mathrm{x} 10^{\wedge} 4$ ) and equivalent isotropic displacement parameters $\left(\mathrm{A}^{\wedge} 2 \times 10^{\wedge} 3\right) . \mathrm{U}(\mathrm{eq})$ is defined as one third of the trace of the orthogonalized Uij tensor.

\begin{tabular}{lllll}
\hline & $\mathrm{x}$ & $\mathrm{y}$ & $\mathrm{z}$ & $\mathrm{U}(\mathrm{eq})$ \\
\hline $\mathrm{Zn}(1)$ & $8514(1)$ & $4575(1)$ & $1188(1)$ & $65(1)$ \\
$\mathrm{N}(1)$ & $10015(8)$ & $3720(4)$ & $1734(3)$ & $53(2)$ \\
$\mathrm{N}(2)$ & $9213(8)$ & $5695(4)$ & $1773(3)$ & $51(2)$ \\
$\mathrm{C}(1)$ & $11345(9)$ & $3073(6)$ & $2766(4)$ & $57(2)$ \\
$\mathrm{C}(2)$ & $10501(7)$ & $3860(5)$ & $2374(3)$ & $39(2)$ \\
$\mathrm{C}(3)$ & $10215(7)$ & $4704(5)$ & $2686(3)$ & $38(2)$ \\
$\mathrm{C}(4)$ & $9723(8)$ & $5601(5)$ & $2409(3)$ & $40(2)$ \\
$\mathrm{C}(5)$ & $9848(10)$ & $6446(6)$ & $2856(4)$ & $62(2)$ \\
$\mathrm{C}(6)$ & $8852(9)$ & $6610(5)$ & $1493(3)$ & $44(2)$ \\
$\mathrm{C}(7)$ & $7471(9)$ & $7028(6)$ & $1516(4)$ & $54(2)$ \\
$\mathrm{C}(8)$ & $7061(8)$ & $7851(5)$ & $1165(3)$ & $46(2)$
\end{tabular}




\begin{tabular}{|c|c|c|c|c|}
\hline $\mathrm{C}(9)$ & 7998(7) & $8299(5)$ & 791(3) & $40(2)$ \\
\hline$C(10)$ & $9458(8)$ & $7881(5)$ & $780(3)$ & $44(2)$ \\
\hline $\mathrm{C}(11)$ & $9856(8)$ & $7051(5)$ & $1129(3)$ & $49(2)$ \\
\hline$C(12)$ & $7577(7)$ & $9182(5)$ & 418(3) & $38(1)$ \\
\hline $\mathrm{C}(13)$ & $7820(7)$ & $9326(4)$ & $-236(3)$ & $38(2)$ \\
\hline$C(14)$ & 7491(7) & 10191(5) & $-525(3)$ & $42(2)$ \\
\hline$C(15)$ & $6856(8)$ & $10926(5)$ & $-212(4)$ & $50(2)$ \\
\hline$C(16)$ & $6552(8)$ & $10787(5)$ & 411(4) & $49(2)$ \\
\hline $\mathrm{C}(17)$ & $6897(8)$ & $9939(6)$ & $721(4)$ & $49(2)$ \\
\hline$C(18)$ & $8384(7)$ & $8538(5)$ & $-640(3)$ & $38(1)$ \\
\hline C(19) & $7667(8)$ & $7637(5)$ & $-698(4)$ & $49(2)$ \\
\hline $\mathrm{C}(20)$ & 8190(9) & $6938(5)$ & $-071(4)$ & $56(2)$ \\
\hline $\mathrm{C}(21)$ & $9416(9)$ & $7073(5)$ & $-1396(3)$ & $47(2)$ \\
\hline $\mathrm{C}(22)$ & $10108(9)$ & 7957(6) & $-1342(4)$ & $56(2)$ \\
\hline$C(23)$ & $9614(9)$ & $8674(5)$ & $-970(3)$ & $51(2)$ \\
\hline $\mathrm{N}(3)$ & $6372(9)$ & $4433(5)$ & $1656(4)$ & $68(2)$ \\
\hline$C(24)$ & $7965(14)$ & $4469(8)$ & $245(4)$ & $91(3)$ \\
\hline$C(26)$ & $6326(11)$ & $4180(8)$ & $2281(5)$ & $83(3)$ \\
\hline $\mathrm{C}(25)$ & $6927(18)$ & $3534(9)$ & $73(5)$ & $127(5)$ \\
\hline $\mathrm{C}(27)$ & $5257(13)$ & $4429(10)$ & 2631(6) & $100(4)$ \\
\hline $\mathrm{C}(28)$ & $4074(11)$ & 4987(9) & $2354(7)$ & $90(3)$ \\
\hline$C(29)$ & $4026(12)$ & $5239(7)$ & $1729(6)$ & $85(3)$ \\
\hline $\mathrm{C}(30)$ & $5179(13)$ & 4941(8) & $1415(5)$ & $85(3)$ \\
\hline
\end{tabular}

Table 2. Bond lengths [A] and angles [deg].

\begin{tabular}{llll}
\hline $\mathrm{Zn}(1)-\mathrm{C}(24)$ & $1.949(9)$ & $\mathrm{Zn}(1)-\mathrm{N}(1)$ & $2.021(6)$ \\
$\mathrm{Zn}(1)-\mathrm{N}(2)$ & $2.022(6)$ & $\mathrm{Zn}(1)-\mathrm{N}(3)$ & $2.267(8)$ \\
$\mathrm{N}(1)-\mathrm{C}(2)$ & $1.348(8)$ & $\mathrm{N}(1)-\mathrm{C}(21) \# 1$ & $1.438(9)$ \\
$\mathrm{N}(2)-\mathrm{C}(4)$ & $1.337(8)$ & $\mathrm{N}(2)-\mathrm{C}(6)$ & $1.422(9)$ \\
$\mathrm{C}(1)-\mathrm{C}(2)$ & $1.505(9)$ & $\mathrm{C}(2)-\mathrm{C}(3)$ & $1.385(9)$ \\
$\mathrm{C}(3)-\mathrm{C}(4)$ & $1.421(9)$ & $\mathrm{C}(4)-\mathrm{C}(5)$ & $1.493(9)$ \\
$\mathrm{C}(6)-\mathrm{C}(7)$ & $1.372(11)$ & $\mathrm{C}(6)-\mathrm{C}(11)$ & $1.391(10)$ \\
$\mathrm{C}(7)-\mathrm{C}(8)$ & $1.381(10)$ & $\mathrm{C}(8)-\mathrm{C}(9)$ & $1.367(9)$ \\
$\mathrm{C}(9)-\mathrm{C}(10)$ & $1.433(10)$ & $\mathrm{C}(9)-\mathrm{C}(12)$ & $1.475(9)$
\end{tabular}




\begin{tabular}{|c|c|c|c|}
\hline $\mathrm{C}(10)-\mathrm{C}(11)$ & $1.384(10)$ & $\mathrm{C}(12)-\mathrm{C}(17)$ & $1.407(10)$ \\
\hline$C(12)-C(13)$ & $1.412(9)$ & $C(13)-C(14)$ & $1.362(9)$ \\
\hline $\mathrm{C}(13)-\mathrm{C}(18)$ & $1.508(9)$ & $\mathrm{C}(14)-\mathrm{C}(15)$ & $1.376(10)$ \\
\hline $\mathrm{C}(15)-\mathrm{C}(16)$ & $1.364(10)$ & $\mathrm{C}(16)-\mathrm{C}(17)$ & $1.361(11)$ \\
\hline $\mathrm{C}(18)-\mathrm{C}(23)$ & $1.383(10)$ & $\mathrm{C}(18)-\mathrm{C}(19)$ & $1.409(9)$ \\
\hline $\mathrm{C}(19)-\mathrm{C}(20)$ & $1.365(10)$ & $C(20)-C(21)$ & $1.374(11)$ \\
\hline$C(21)-C(22)$ & $1.377(11)$ & $\mathrm{C}(21)-\mathrm{N}(1) \# 1$ & $1.438(9)$ \\
\hline$C(22)-C(23)$ & $1.372(10)$ & $\mathrm{N}(3)-\mathrm{C}(30)$ & $1.320(12)$ \\
\hline $\mathrm{N}(3)-\mathrm{C}(26)$ & $1.346(12)$ & $C(24)-C(25)$ & $1.613(15)$ \\
\hline$C(26)-C(27)$ & $1.320(15)$ & $\mathrm{C}(27)-\mathrm{C}(28)$ & $1.374(16)$ \\
\hline $\mathrm{C}(28)-\mathrm{C}(29)$ & $1.334(15)$ & $C(29)-C(30)$ & $1.356(15)$ \\
\hline $\mathrm{C}(24)-\mathrm{Zn}(1)-\mathrm{N}(1)$ & $124.8(4)$ & $\mathrm{C}(24)-\mathrm{Zn}(1)-\mathrm{N}(2)$ & $132.2(4)$ \\
\hline $\mathrm{N}(1)-\mathrm{Zn}(1)-\mathrm{N}(2)$ & $89.8(2)$ & $\mathrm{C}(24)-\mathrm{Zn}(1)-\mathrm{N}(3)$ & $107.9(4)$ \\
\hline $\mathrm{N}(1)-\mathrm{Zn}(1)-\mathrm{N}(3)$ & $104.2(3)$ & $\mathrm{N}(2)-\mathrm{Zn}(1)-\mathrm{N}(3)$ & $91.3(3)$ \\
\hline $\mathrm{C}(2)-\mathrm{N}(1)-\mathrm{C}(21) \# 1$ & $120.0(6)$ & $\mathrm{C}(2)-\mathrm{N}(1)-\mathrm{Zn}(1)$ & $124.2(4)$ \\
\hline $\mathrm{C}(21) \# 1-\mathrm{N}(1)-\mathrm{Zn}(1)$ & $115.8(4)$ & $\mathrm{C}(4)-\mathrm{N}(2)-\mathrm{C}(6)$ & $121.1(5)$ \\
\hline $\mathrm{C}(4)-\mathrm{N}(2)-\mathrm{Zn}(1)$ & $123.2(4)$ & $\mathrm{C}(6)-\mathrm{N}(2)-\mathrm{Zn}(1)$ & $114.6(4)$ \\
\hline $\mathrm{N}(1)-\mathrm{C}(2)-\mathrm{C}(3)$ & $121.7(6)$ & $\mathrm{N}(1)-\mathrm{C}(2)-\mathrm{C}(1)$ & $119.6(6)$ \\
\hline $\mathrm{C}(3)-\mathrm{C}(2)-\mathrm{C}(1)$ & $118.8(6)$ & $\mathrm{C}(2)-\mathrm{C}(3)-\mathrm{C}(4)$ & $129.0(6)$ \\
\hline $\mathrm{N}(2)-\mathrm{C}(4)-\mathrm{C}(3)$ & $121.8(6)$ & $\mathrm{N}(2)-\mathrm{C}(4)-\mathrm{C}(5)$ & 121.1(6) \\
\hline$C(3)-C(4)-C(5)$ & $117.0(6)$ & $C(7)-C(6)-C(11)$ & $118.7(7)$ \\
\hline $\mathrm{C}(7)-\mathrm{C}(6)-\mathrm{N}(2)$ & $121.6(7)$ & $\mathrm{C}(11)-\mathrm{C}(6)-\mathrm{N}(2)$ & $119.4(7)$ \\
\hline $\mathrm{C}(6)-\mathrm{C}(7)-\mathrm{C}(8)$ & $120.7(7)$ & $\mathrm{C}(9)-\mathrm{C}(8)-\mathrm{C}(7)$ & $122.6(7)$ \\
\hline $\mathrm{C}(8)-\mathrm{C}(9)-\mathrm{C}(10)$ & $116.8(6)$ & $\mathrm{C}(8)-\mathrm{C}(9)-\mathrm{C}(12)$ & $123.1(6)$ \\
\hline$C(10)-C(9)-C(12)$ & $120.1(6)$ & $\mathrm{C}(11)-\mathrm{C}(10)-\mathrm{C}(9)$ & $120.2(6)$ \\
\hline $\mathrm{C}(10)-\mathrm{C}(11)-\mathrm{C}(6)$ & $120.9(7)$ & $\mathrm{C}(17)-\mathrm{C}(12)-\mathrm{C}(13)$ & $116.8(6)$ \\
\hline $\mathrm{C}(17)-\mathrm{C}(12)-\mathrm{C}(9)$ & $119.4(6)$ & $\mathrm{C}(13)-\mathrm{C}(12)-\mathrm{C}(9)$ & $123.8(6)$ \\
\hline $\mathrm{C}(14)-\mathrm{C}(13)-\mathrm{C}(12)$ & $119.5(6)$ & $\mathrm{C}(14)-\mathrm{C}(13)-\mathrm{C}(18)$ & $118.3(6)$ \\
\hline $\mathrm{C}(12)-\mathrm{C}(13)-\mathrm{C}(18)$ & $122.2(6)$ & $\mathrm{C}(13)-\mathrm{C}(14)-\mathrm{C}(15)$ & $122.2(7)$ \\
\hline$C(16)-C(15)-C(14)$ & $119.0(7)$ & $\mathrm{C}(17)-\mathrm{C}(16)-\mathrm{C}(15)$ & $120.4(7)$ \\
\hline$C(16)-C(17)-C(12)$ & $121.9(7)$ & $\mathrm{C}(23)-\mathrm{C}(18)-\mathrm{C}(19)$ & $117.6(6)$ \\
\hline $\mathrm{C}(23)-\mathrm{C}(18)-\mathrm{C}(13)$ & $121.3(6)$ & $\mathrm{C}(19)-\mathrm{C}(18)-\mathrm{C}(13)$ & 121.1(6) \\
\hline $\mathrm{C}(20)-\mathrm{C}(19)-\mathrm{C}(18)$ & $120.0(7)$ & $\mathrm{C}(19)-\mathrm{C}(20)-\mathrm{C}(21)$ & $122.3(7)$ \\
\hline $\mathrm{C}(20)-\mathrm{C}(21)-\mathrm{C}(22)$ & $117.6(7)$ & $\mathrm{C}(20)-\mathrm{C}(21)-\mathrm{N}(1) \# 1$ & $119.3(7)$ \\
\hline $\mathrm{C}(22)-\mathrm{C}(21)-\mathrm{N}(1) \# 1$ & $122.9(7)$ & $\mathrm{C}(23)-\mathrm{C}(22)-\mathrm{C}(21)$ & $121.6(7)$ \\
\hline $\mathrm{C}(22)-\mathrm{C}(23)-\mathrm{C}(18)$ & $121.0(7)$ & $\mathrm{C}(30)-\mathrm{N}(3)-\mathrm{C}(26)$ & $112.0(9)$ \\
\hline
\end{tabular}




$\begin{array}{llll}\mathrm{C}(30)-\mathrm{N}(3)-\mathrm{Zn}(1) & 118.5(7) & \mathrm{C}(26)-\mathrm{N}(3)-\mathrm{Zn}(1) & 124.8(6) \\ \mathrm{C}(25)-\mathrm{C}(24)-\mathrm{Zn}(1) & 109.9(7) & \mathrm{C}(27)-\mathrm{C}(26)-\mathrm{N}(3) & 125.2(10) \\ \mathrm{C}(26)-\mathrm{C}(27)-\mathrm{C}(28) & 119.6(11) & \mathrm{C}(29)-\mathrm{C}(28)-\mathrm{C}(27) & 118.3(11) \\ \mathrm{C}(28)-\mathrm{C}(29)-\mathrm{C}(30) & 117.2(10) & \mathrm{N}(3)-\mathrm{C}(30)-\mathrm{C}(29) & 127.7(11)\end{array}$

Symmetry transformations used to generate equivalent atoms:

Table 3. Anisotropic displacement parameters $\left(\mathrm{A}^{\wedge} 2 \times 10^{\wedge} 3\right)$. The anisotropic displacement factor exponent takes the form: $-2 \mathrm{pi}^{\wedge} 2\left[\mathrm{~h}^{\wedge} 2 \mathrm{a}^{* \wedge} 2 \mathrm{U} 11+\ldots+2 \mathrm{~h} \mathrm{k} \mathrm{a}^{*} \mathrm{~b}^{*}\right.$ U12 ]

\begin{tabular}{|c|c|c|c|c|c|c|}
\hline & U11 & U22 & U33 & $\mathrm{U} 23$ & U13 & U12 \\
\hline $\mathrm{Zn}(1)$ & $88(1)$ & $50(1)$ & $54(1)$ & $-3(1)$ & $-2(1)$ & $6(1)$ \\
\hline $\mathrm{N}(1)$ & $92(5)$ & $33(3)$ & $34(3)$ & $-3(2)$ & $13(3)$ & $15(3)$ \\
\hline $\mathrm{N}(2)$ & $89(5)$ & $29(3)$ & $36(3)$ & $-5(2)$ & $14(3)$ & $-1(3)$ \\
\hline $\mathrm{C}(1)$ & $64(5)$ & $54(5)$ & $50(4)$ & $2(4)$ & $2(4)$ & $19(4)$ \\
\hline $\mathrm{C}(2)$ & $44(4)$ & $39(3)$ & $37(3)$ & $7(3)$ & $12(3)$ & $-2(3)$ \\
\hline $\mathrm{C}(3)$ & $48(4)$ & $42(4)$ & $27(3)$ & $-2(3)$ & $10(3)$ & $4(3)$ \\
\hline $\mathrm{C}(4)$ & $44(4)$ & $42(4)$ & $33(3)$ & $-3(3)$ & $8(3)$ & $2(3)$ \\
\hline$C(5)$ & $96(6)$ & $43(4)$ & $44(4)$ & $-12(3)$ & $0(4)$ & $12(4)$ \\
\hline$C(6)$ & $68(5)$ & $33(3)$ & $30(3)$ & $-3(3)$ & $6(3)$ & $-4(3)$ \\
\hline$C(7)$ & $62(5)$ & $48(4)$ & $56(4)$ & $3(4)$ & $18(4)$ & $-13(4)$ \\
\hline $\mathrm{C}(8)$ & 41(4) & $47(4)$ & $52(4)$ & $6(3)$ & $14(3)$ & $3(3)$ \\
\hline $\mathrm{C}(9)$ & $47(4)$ & $38(3)$ & $33(3)$ & $-3(3)$ & $0(3)$ & $-3(3)$ \\
\hline $\mathrm{C}(10)$ & $46(4)$ & $41(4)$ & $45(4)$ & $7(3)$ & $4(3)$ & $-9(3)$ \\
\hline$C(11)$ & $40(4)$ & $50(4)$ & $53(4)$ & $5(3)$ & $-2(3)$ & $6(3)$ \\
\hline$C(12)$ & $33(3)$ & $38(3)$ & $42(4)$ & $-4(3)$ & $7(3)$ & $-3(3)$ \\
\hline$C(13)$ & $35(3)$ & $31(3)$ & $45(4)$ & $-7(3)$ & $0(3)$ & $1(3)$ \\
\hline$C(14)$ & $45(4)$ & $41(4)$ & $37(3)$ & $-3(3)$ & $-3(3)$ & $5(3)$ \\
\hline $\mathrm{C}(15)$ & $50(4)$ & $37(4)$ & $61(5)$ & $-2(3)$ & $-4(4)$ & $5(3)$ \\
\hline$C(16)$ & $43(4)$ & $40(4)$ & $64(5)$ & $-11(3)$ & $8(3)$ & $5(3)$ \\
\hline $\mathrm{C}(17)$ & $45(4)$ & $57(5)$ & $47(4)$ & $-10(4)$ & $13(3)$ & $-5(4)$ \\
\hline$C(18)$ & $45(4)$ & $33(3)$ & $35(3)$ & $-3(3)$ & $0(3)$ & $-1(3)$ \\
\hline$C(19)$ & $50(4)$ & $42(4)$ & $56(4)$ & $-9(3)$ & $10(3)$ & $-5(3)$ \\
\hline$C(20)$ & $69(5)$ & $32(4)$ & $66(5)$ & $-13(3)$ & $2(4)$ & $-7(4)$ \\
\hline
\end{tabular}




\begin{tabular}{lllllll}
$\mathrm{C}(21)$ & $63(5)$ & $45(4)$ & $33(3)$ & $0(3)$ & $1(3)$ & $10(4)$ \\
$\mathrm{C}(22)$ & $66(5)$ & $46(4)$ & $61(5)$ & $-3(4)$ & $24(4)$ & $1(4)$ \\
$\mathrm{C}(23)$ & $65(5)$ & $38(4)$ & $53(4)$ & $-7(3)$ & $19(4)$ & $-4(4)$ \\
$\mathrm{N}(3)$ & $79(5)$ & $56(4)$ & $61(4)$ & $5(3)$ & $-17(4)$ & $-6(4)$ \\
$\mathrm{C}(24)$ & $126(9)$ & $91(8)$ & $50(5)$ & $-1(5)$ & $-7(5)$ & $-13(7)$ \\
$\mathrm{C}(26)$ & $65(6)$ & $77(7)$ & $101(8)$ & $30(6)$ & $-14(6)$ & $-14(5)$ \\
$\mathrm{C}(25)$ & $233(16)$ & $76(7)$ & $58(6)$ & $-4(5)$ & $-31(8)$ & $-32(9)$ \\
$\mathrm{C}(27)$ & $70(7)$ & $151(12)$ & $79(7)$ & $36(7)$ & $12(6)$ & $-26(7)$ \\
$\mathrm{C}(28)$ & $58(6)$ & $101(8)$ & $113(9)$ & $-9(8)$ & $20(6)$ & $-10(6)$ \\
$\mathrm{C}(29)$ & $67(6)$ & $62(6)$ & $120(10)$ & $16(6)$ & $-14(6)$ & $-5(5)$ \\
$\mathrm{C}(30)$ & $94(7)$ & $67(6)$ & $86(7)$ & $6(5)$ & $-14(6)$ & $9(6)$ \\
& & & & & & \\
\hline
\end{tabular}

Table 4. Hydrogen coordinates $\left(x 10^{\wedge} 4\right)$ and isotropic displacement parameters $\left(\mathrm{A}^{\wedge} 2\right.$ x 10^3).

\begin{tabular}{|c|c|c|c|c|}
\hline & $\mathrm{x}$ & $\mathrm{y}$ & $\mathrm{z}$ & $\mathrm{U}(\mathrm{eq})$ \\
\hline $\mathrm{H}(1 \mathrm{~A})$ & 10729 & 2508 & 2745 & 85 \\
\hline $\mathrm{H}(1 \mathrm{~B})$ & 11582 & 3274 & 3213 & 85 \\
\hline $\mathrm{H}(1 \mathrm{C})$ & 12263 & 2936 & 2591 & 85 \\
\hline $\mathrm{H}(3)$ & 10364 & 4681 & 3140 & 46 \\
\hline $\mathrm{H}(5 \mathrm{~A})$ & 10455 & 6931 & 2689 & 93 \\
\hline $\mathrm{H}(5 \mathrm{~B})$ & 10311 & 6254 & 3283 & 93 \\
\hline $\mathrm{H}(5 \mathrm{C})$ & 8858 & 6698 & 2883 & 93 \\
\hline $\mathrm{H}(7)$ & 6804 & 6754 & 1771 & 65 \\
\hline $\mathrm{H}(8)$ & 6110 & 8111 & 1184 & 55 \\
\hline $\mathrm{H}(10)$ & 10141 & 8167 & 539 & 53 \\
\hline $\mathrm{H}(11)$ & 10807 & 6786 & 1120 & 58 \\
\hline $\mathrm{H}(14)$ & 7702 & 10289 & -949 & 50 \\
\hline $\mathrm{H}(15)$ & 6637 & 11509 & -421 & 60 \\
\hline $\mathrm{H}(16)$ & 6106 & 11274 & 625 & 59 \\
\hline $\mathrm{H}(17)$ & 6677 & 9858 & 1144 & 59 \\
\hline $\mathrm{H}(19)$ & 6838 & 7519 & -483 & 59 \\
\hline $\mathrm{H}(20)$ & 7698 & 6349 & -1107 & 68 \\
\hline $\mathrm{H}(22)$ & 10929 & 8070 & -1564 & 68 \\
\hline
\end{tabular}




\begin{tabular}{lcccc}
$\mathrm{H}(23)$ & 10112 & 9260 & -939 & 61 \\
$\mathrm{H}(24 \mathrm{~A})$ & 7418 & 5037 & 79 & 109 \\
$\mathrm{H}(24 \mathrm{~B})$ & 8872 & 4421 & 37 & 109 \\
$\mathrm{H}(26)$ & 7110 & 3801 & 2483 & 100 \\
$\mathrm{H}(25 \mathrm{~A})$ & 7319 & 3011 & 347 & 191 \\
$\mathrm{H}(25 \mathrm{~B})$ & 6932 & 3365 & -377 & 191 \\
$\mathrm{H}(25 \mathrm{C})$ & 5911 & 3670 & 147 & 191 \\
$\mathrm{H}(27)$ & 5305 & 4227 & 3062 & 120 \\
$\mathrm{H}(28)$ & 3325 & 5184 & 2596 & 108 \\
$\mathrm{H}(29)$ & 3236 & 5604 & 1517 & 103 \\
$\mathrm{H}(30)$ & 5123 & 5115 & 977 & 102 \\
\hline
\end{tabular}




\section{X-Ray Report for 7}

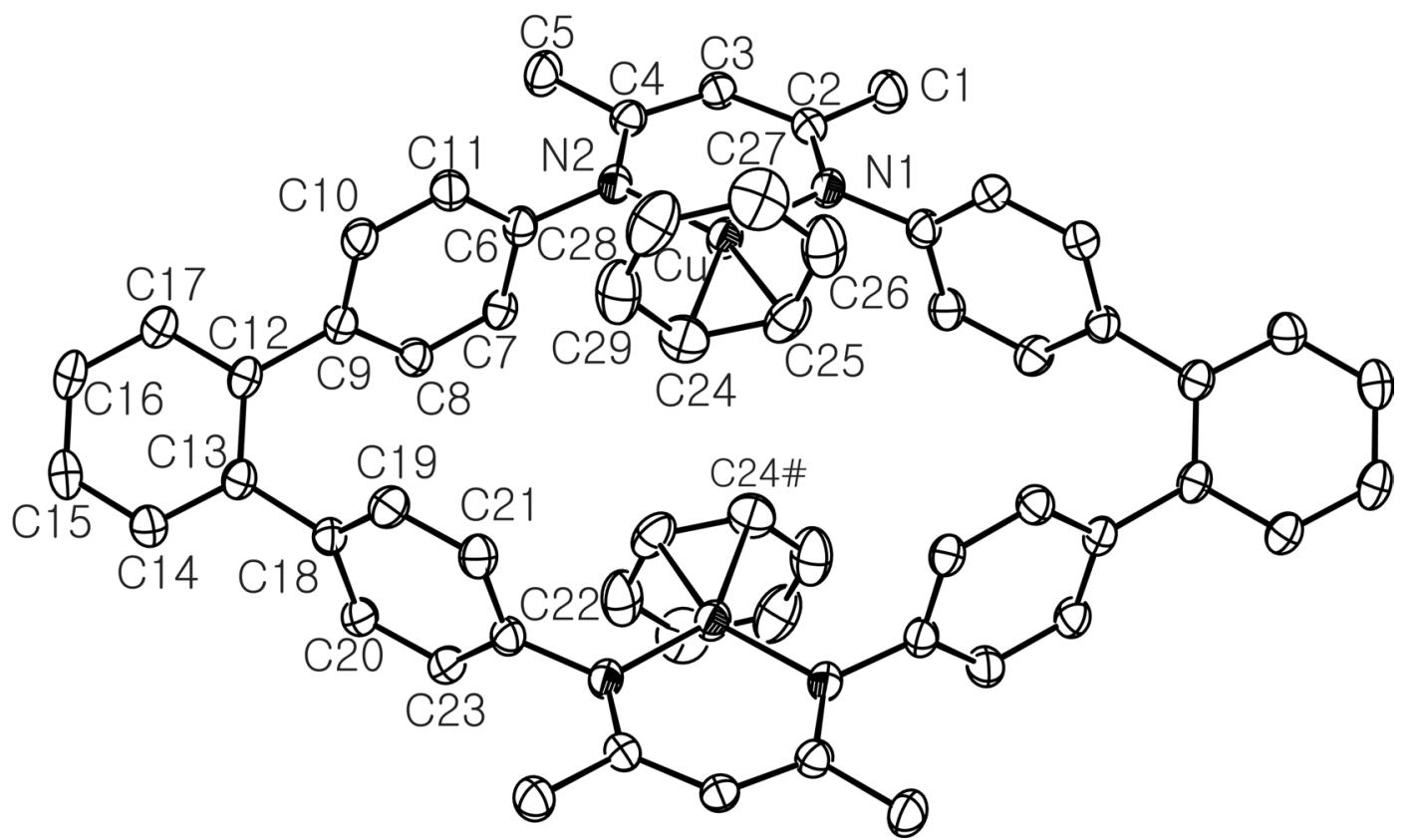

Table 1. Atomic coordinates ( $\left.\mathrm{x} 10^{\wedge} 4\right)$ and equivalent isotropic displacement parameters $\left(\mathrm{A}^{\wedge} 2 \times 10^{\wedge} 3\right) . \mathrm{U}(\mathrm{eq})$ is defined as one third of the trace of the orthogonalized Uij tensor.

\begin{tabular}{lllll}
\hline & $\mathrm{x}$ & $\mathrm{y}$ & $\mathrm{z}$ & $\mathrm{U}(\mathrm{eq})$ \\
\hline $\mathrm{Cu}(1)$ & $5944(1)$ & $5492(1)$ & $2045(1)$ & $48(1)$ \\
$\mathrm{N}(1)$ & $4623(3)$ & $4884(2)$ & $2888(2)$ & $46(1)$ \\
$\mathrm{N}(2)$ & $5988(3)$ & $7099(2)$ & $2503(2)$ & $45(1)$ \\
$\mathrm{C}(1)$ & $3136(4)$ & $5059(3)$ & $4269(3)$ & $58(1)$ \\
$\mathrm{C}(3)$ & $4630(3)$ & $6734(2)$ & $3873(2)$ & $47(1)$ \\
$\mathrm{C}(2)$ & $4179(3)$ & $5554(2)$ & $3630(2)$ & $44(1)$ \\
$\mathrm{C}(4)$ & $5409(3)$ & $7463(2)$ & $3339(2)$ & $44(1)$ \\
$\mathrm{C}(5)$ & $5578(4)$ & $8724(3)$ & $3735(3)$ & $61(1)$ \\
$\mathrm{C}(6)$ & $6467(3)$ & $7913(2)$ & $1849(2)$ & $43(1)$ \\
$\mathrm{C}(7)$ & $5507(3)$ & $8127(2)$ & $971(2)$ & $44(1)$ \\
$\mathrm{C}(8)$ & $5921(3)$ & $8833(2)$ & $270(2)$ & $45(1)$ \\
$\mathrm{C}(9)$ & $7313(3)$ & $9372(2)$ & $424(2)$ & $41(1)$ \\
$\mathrm{C}(10)$ & $8261(3)$ & $9162(3)$ & $1311(2)$ & $49(1)$
\end{tabular}




\begin{tabular}{|c|c|c|c|c|}
\hline$C(11)$ & $7855(3)$ & $8435(3)$ & $2010(3)$ & $51(1)$ \\
\hline $\mathrm{C}(12)$ & $7726(3)$ & 10157(2) & $-340(2)$ & $42(1)$ \\
\hline$C(13)$ & 7497(3) & $9815(2)$ & $-1447(2)$ & $43(1)$ \\
\hline $\mathrm{C}(14)$ & 7751(3) & $10625(3)$ & $-2118(3)$ & $52(1)$ \\
\hline$C(15)$ & $8219(4)$ & $11757(3)$ & $-1718(3)$ & $58(1)$ \\
\hline$C(16)$ & 8498(3) & 12087(3) & $-632(3)$ & $55(1)$ \\
\hline $\mathrm{C}(17)$ & $8251(3)$ & $11292(2)$ & $50(3)$ & $48(1)$ \\
\hline$C(18)$ & 7003(3) & $8591(2)$ & $-1893(2)$ & $42(1)$ \\
\hline C(19) & 7714(3) & $7715(3)$ & $-1508(2)$ & $48(1)$ \\
\hline$C(20)$ & $5805(4)$ & $8290(3)$ & $-2675(2)$ & $52(1)$ \\
\hline $\mathrm{C}(21)$ & 2782(3) & $3421(3)$ & $1864(2)$ & $48(1)$ \\
\hline $\mathrm{C}(22)$ & 4015(3) & $3709(2)$ & $2621(2)$ & $44(1)$ \\
\hline $\mathrm{C}(23)$ & 4713(4) & $2842(3)$ & $3027(2)$ & $52(1)$ \\
\hline $\mathrm{C}(24)$ & 7058(4) & $5146(3)$ & $780(3)$ & $66(1)$ \\
\hline$C(25)$ & $6650(4)$ & $4141(3)$ & $1184(3)$ & $66(1)$ \\
\hline$C(26)$ & $7669(5)$ & $3701(3)$ & 1904(4) & $80(1)$ \\
\hline $\mathrm{C}(27)$ & $9027(5)$ & $4221(4)$ & $2176(4)$ & $91(1)$ \\
\hline $\mathrm{C}(28)$ & $9435(5)$ & $5202(4)$ & $1743(4)$ & $88(1)$ \\
\hline C(29) & $8469(5)$ & $5652(4)$ & 1068(4) & $81(1)$ \\
\hline$C(30)$ & 8933(6) & $7808(5)$ & $-4339(6)$ & $109(2)$ \\
\hline C(31) & $9219(8)$ & $7244(5)$ & $-5220(5)$ & 116(2) \\
\hline C(32) & $10589(11)$ & $7437(7)$ & $-5459(5)$ & $134(2)$ \\
\hline $\mathrm{C}(33)$ & $11558(7)$ & $8230(6)$ & $-4792(7)$ & $136(2)$ \\
\hline$C(34)$ & $11215(7)$ & $8749(5)$ & $-3904(6)$ & $145(2)$ \\
\hline $\mathrm{C}(35)$ & 9912(8) & $8542(5)$ & $-3697(6)$ & $133(2)$ \\
\hline
\end{tabular}

Table 2. Bond lengths [A] and angles [deg].

\begin{tabular}{llll}
\hline $\mathrm{Cu}(1)-\mathrm{N}(2)$ & $1.925(2)$ & $\mathrm{Cu}(1)-\mathrm{N}(1)$ & $1.935(2)$ \\
$\mathrm{Cu}(1)-\mathrm{C}(25)$ & $2.078(3)$ & $\mathrm{Cu}(1)-\mathrm{C}(24)$ & $2.112(3)$ \\
$\mathrm{N}(1)-\mathrm{C}(2)$ & $1.325(4)$ & $\mathrm{N}(1)-\mathrm{C}(22)$ & $1.436(4)$ \\
$\mathrm{N}(2)-\mathrm{C}(4)$ & $1.337(4)$ & $\mathrm{N}(2)-\mathrm{C}(6)$ & $1.435(4)$ \\
$\mathrm{C}(1)-\mathrm{C}(2)$ & $1.509(4)$ & $\mathrm{C}(3)-\mathrm{C}(4)$ & $1.395(4)$ \\
$\mathrm{C}(3)-\mathrm{C}(2)$ & $1.406(4)$ & $\mathrm{C}(4)-\mathrm{C}(5)$ & $1.509(4)$ \\
$\mathrm{C}(6)-\mathrm{C}(11)$ & $1.385(4)$ & $\mathrm{C}(6)-\mathrm{C}(7)$ & $1.390(4)$
\end{tabular}




\begin{tabular}{|c|c|c|c|}
\hline$C(7)-C(8)$ & $1.371(4)$ & $\mathrm{C}(8)-\mathrm{C}(9)$ & $1.396(4)$ \\
\hline $\mathrm{C}(9)-\mathrm{C}(10)$ & $1.390(4)$ & $\mathrm{C}(9)-\mathrm{C}(12)$ & $1.494(4)$ \\
\hline $\mathrm{C}(10)-\mathrm{C}(11)$ & $1.382(4)$ & $\mathrm{C}(12)-\mathrm{C}(17)$ & $1.395(4)$ \\
\hline $\mathrm{C}(12)-\mathrm{C}(13)$ & $1.404(4)$ & $\mathrm{C}(13)-\mathrm{C}(14)$ & $1.385(4)$ \\
\hline $\mathrm{C}(13)-\mathrm{C}(18)$ & $1.498(4)$ & $\mathrm{C}(14)-\mathrm{C}(15)$ & $1.384(4)$ \\
\hline $\mathrm{C}(15)-\mathrm{C}(16)$ & $1.375(5)$ & $\mathrm{C}(16)-\mathrm{C}(17)$ & $1.381(4)$ \\
\hline C(18)-C(20) & $1.379(4)$ & $\mathrm{C}(18)-\mathrm{C}(19)$ & $1.387(4)$ \\
\hline C(19)-C(21)\#1 & $1.384(4)$ & $\mathrm{C}(20)-\mathrm{C}(23) \# 1$ & $1.382(4)$ \\
\hline $\mathrm{C}(21)-\mathrm{C}(22)$ & $1.383(4)$ & $\mathrm{C}(21)-\mathrm{C}(19) \# 1$ & $1.384(4)$ \\
\hline $\mathrm{C}(22)-\mathrm{C}(23)$ & $1.381(4)$ & $\mathrm{C}(23)-\mathrm{C}(20) \# 1$ & $1.382(4)$ \\
\hline $\mathrm{C}(24)-\mathrm{C}(25)$ & $1.397(5)$ & $\mathrm{C}(24)-\mathrm{C}(29)$ & $1.397(5)$ \\
\hline$C(25)-C(26)$ & $1.406(6)$ & $\mathrm{C}(26)-\mathrm{C}(27)$ & $1.357(6)$ \\
\hline $\mathrm{C}(27)-\mathrm{C}(28)$ & $1.391(6)$ & $\mathrm{C}(28)-\mathrm{C}(29)$ & $1.347(6)$ \\
\hline $\mathrm{C}(30)-\mathrm{C}(35)$ & $1.318(7)$ & $\mathrm{C}(30)-\mathrm{C}(31)$ & $1.331(8)$ \\
\hline $\mathrm{C}(31)-\mathrm{C}(32)$ & $1.403(9)$ & $\mathrm{C}(32)-\mathrm{C}(33)$ & $1.365(8)$ \\
\hline $\mathrm{C}(33)-\mathrm{C}(34)$ & $1.339(8)$ & $\mathrm{C}(34)-\mathrm{C}(35)$ & $1.327(7)$ \\
\hline $\mathrm{N}(2)-\mathrm{Cu}(1)-\mathrm{N}(1)$ & $99.40(10)$ & $\mathrm{N}(2)-\mathrm{Cu}(1)-\mathrm{C}(25)$ & $151.52(13)$ \\
\hline $\mathrm{N}(1)-\mathrm{Cu}(1)-\mathrm{C}(25)$ & 108.98(13) & $\mathrm{N}(2)-\mathrm{Cu}(1)-\mathrm{C}(24)$ & $113.45(13)$ \\
\hline $\mathrm{N}(1)-\mathrm{Cu}(1)-\mathrm{C}(24)$ & $146.46(13)$ & $\mathrm{C}(25)-\mathrm{Cu}(1)-\mathrm{C}(24)$ & $38.93(14)$ \\
\hline $\mathrm{C}(2)-\mathrm{N}(1)-\mathrm{C}(22)$ & $120.0(3)$ & $\mathrm{C}(2)-\mathrm{N}(1)-\mathrm{Cu}(1)$ & $121.16(19)$ \\
\hline $\mathrm{C}(22)-\mathrm{N}(1)-\mathrm{Cu}(1)$ & 118.53(19) & $\mathrm{C}(4)-\mathrm{N}(2)-\mathrm{C}(6)$ & $119.3(2)$ \\
\hline $\mathrm{C}(4)-\mathrm{N}(2)-\mathrm{Cu}(1)$ & $121.0(2)$ & $\mathrm{C}(6)-\mathrm{N}(2)-\mathrm{Cu}(1)$ & $119.13(19)$ \\
\hline $\mathrm{C}(4)-\mathrm{C}(3)-\mathrm{C}(2)$ & $130.1(3)$ & $\mathrm{N}(1)-\mathrm{C}(2)-\mathrm{C}(3)$ & $123.5(3)$ \\
\hline $\mathrm{N}(1)-\mathrm{C}(2)-\mathrm{C}(1)$ & $120.0(3)$ & $\mathrm{C}(3)-\mathrm{C}(2)-\mathrm{C}(1)$ & $116.5(3)$ \\
\hline $\mathrm{N}(2)-\mathrm{C}(4)-\mathrm{C}(3)$ & $123.5(3)$ & $\mathrm{N}(2)-\mathrm{C}(4)-\mathrm{C}(5)$ & $119.5(3)$ \\
\hline$C(3)-C(4)-C(5)$ & $117.1(3)$ & $\mathrm{C}(11)-\mathrm{C}(6)-\mathrm{C}(7)$ & 118.7(3) \\
\hline $\mathrm{C}(11)-\mathrm{C}(6)-\mathrm{N}(2)$ & $123.3(3)$ & $\mathrm{C}(7)-\mathrm{C}(6)-\mathrm{N}(2)$ & $117.9(3)$ \\
\hline$C(8)-C(7)-C(6)$ & $121.0(3)$ & $\mathrm{C}(7)-\mathrm{C}(8)-\mathrm{C}(9)$ & 121.1(3) \\
\hline $\mathrm{C}(10)-\mathrm{C}(9)-\mathrm{C}(8)$ & $117.5(3)$ & $\mathrm{C}(10)-\mathrm{C}(9)-\mathrm{C}(12)$ & $122.6(3)$ \\
\hline $\mathrm{C}(8)-\mathrm{C}(9)-\mathrm{C}(12)$ & $119.9(3)$ & $\mathrm{C}(11)-\mathrm{C}(10)-\mathrm{C}(9)$ & $121.7(3)$ \\
\hline $\mathrm{C}(10)-\mathrm{C}(11)-\mathrm{C}(6)$ & $120.1(3)$ & $\mathrm{C}(17)-\mathrm{C}(12)-\mathrm{C}(13)$ & $118.8(3)$ \\
\hline $\mathrm{C}(17)-\mathrm{C}(12)-\mathrm{C}(9)$ & 118.9(3) & $C(13)-C(12)-C(9)$ & $122.1(2)$ \\
\hline $\mathrm{C}(14)-\mathrm{C}(13)-\mathrm{C}(12)$ & $118.9(3)$ & $\mathrm{C}(14)-\mathrm{C}(13)-\mathrm{C}(18)$ & $120.6(3)$ \\
\hline $\mathrm{C}(12)-\mathrm{C}(13)-\mathrm{C}(18)$ & $120.5(3)$ & $\mathrm{C}(15)-\mathrm{C}(14)-\mathrm{C}(13)$ & $121.4(3)$ \\
\hline$C(16)-C(15)-C(14)$ & 119.9(3) & $\mathrm{C}(15)-\mathrm{C}(16)-\mathrm{C}(17)$ & $119.5(3)$ \\
\hline$C(16)-C(17)-C(12)$ & $121.4(3)$ & $\mathrm{C}(20)-\mathrm{C}(18)-\mathrm{C}(19)$ & $117.5(3)$ \\
\hline
\end{tabular}




$\begin{array}{llll}\mathrm{C}(20)-\mathrm{C}(18)-\mathrm{C}(13) & 121.6(3) & \mathrm{C}(19)-\mathrm{C}(18)-\mathrm{C}(13) & 120.9(3) \\ \mathrm{C}(21) \# 1-\mathrm{C}(19)-\mathrm{C}(18) & 121.5(3) & \mathrm{C}(18)-\mathrm{C}(20)-\mathrm{C}(23) \# 1 & 121.5(3) \\ \mathrm{C}(22)-\mathrm{C}(21)-\mathrm{C}(19) \# 1 & 120.2(3) & \mathrm{C}(23)-\mathrm{C}(22)-\mathrm{C}(21) & 118.7(3) \\ \mathrm{C}(23)-\mathrm{C}(22)-\mathrm{N}(1) & 121.1(3) & \mathrm{C}(21)-\mathrm{C}(22)-\mathrm{N}(1) & 119.9(3) \\ \mathrm{C}(22)-\mathrm{C}(23)-\mathrm{C}(20) \# 1 & 120.5(3) & \mathrm{C}(25)-\mathrm{C}(24)-\mathrm{C}(29) & 119.2(4) \\ \mathrm{C}(25)-\mathrm{C}(24)-\mathrm{Cu}(1) & 69.2(2) & \mathrm{C}(29)-\mathrm{C}(24)-\mathrm{Cu}(1) & 110.3(3) \\ \mathrm{C}(24)-\mathrm{C}(25)-\mathrm{C}(26) & 118.3(4) & \mathrm{C}(24)-\mathrm{C}(25)-\mathrm{Cu}(1) & 71.9(2) \\ \mathrm{C}(26)-\mathrm{C}(25)-\mathrm{Cu}(1) & 106.8(3) & \mathrm{C}(27)-\mathrm{C}(26)-\mathrm{C}(25) & 120.9(4) \\ \mathrm{C}(26)-\mathrm{C}(27)-\mathrm{C}(28) & 120.3(4) & \mathrm{C}(29)-\mathrm{C}(28)-\mathrm{C}(27) & 119.8(4) \\ \mathrm{C}(28)-\mathrm{C}(29)-\mathrm{C}(24) & 121.4(4) & \mathrm{C}(35)-\mathrm{C}(30)-\mathrm{C}(31) & 120.9(6) \\ \mathrm{C}(30)-\mathrm{C}(31)-\mathrm{C}(32) & 119.1(6) & \mathrm{C}(33)-\mathrm{C}(32)-\mathrm{C}(31) & 118.1(6) \\ \mathrm{C}(34)-\mathrm{C}(33)-\mathrm{C}(32) & 119.9(6) & \mathrm{C}(35)-\mathrm{C}(34)-\mathrm{C}(33) & 120.4(6) \\ \mathrm{C}(30)-\mathrm{C}(35)-\mathrm{C}(34) & 121.5(6) & & \end{array}$

Symmetry transformations used to generate equivalent atoms:

Table 3. Anisotropic displacement parameters $\left(\mathrm{A}^{\wedge} 2 \times 10^{\wedge} 3\right)$. The anisotropic displacement factor exponent takes the form: $-2 \mathrm{pi}^{\wedge} 2\left[\mathrm{~h}^{\wedge} 2 \mathrm{a}^{* \wedge} 2 \mathrm{U} 11+\ldots+2 \mathrm{~h} \mathrm{k} \mathrm{a}^{*} \mathrm{~b}^{*}\right.$ $\mathrm{U} 12$ ]

\begin{tabular}{lllllll}
\hline & $\mathrm{U} 11$ & $\mathrm{U} 2$ & $\mathrm{U} 33$ & $\mathrm{U} 23$ & $\mathrm{U} 13$ & $\mathrm{U} 12$ \\
\hline $\mathrm{Cu}(1)$ & $55(1)$ & $39(1)$ & $52(1)$ & $1(1)$ & $17(1)$ & $0(1)$ \\
$\mathrm{N}(1)$ & $54(2)$ & $36(1)$ & $47(2)$ & $2(1)$ & $15(1)$ & $-2(1)$ \\
$\mathrm{N}(2)$ & $49(2)$ & $37(1)$ & $48(2)$ & $5(1)$ & $11(1)$ & $-1(1)$ \\
$\mathrm{C}(1)$ & $67(2)$ & $52(2)$ & $57(2)$ & $7(2)$ & $23(2)$ & $2(2)$ \\
$\mathrm{C}(3)$ & $62(2)$ & $39(2)$ & $41(2)$ & $0(1)$ & $16(2)$ & $6(2)$ \\
$\mathrm{C}(2)$ & $49(2)$ & $42(2)$ & $41(2)$ & $9(1)$ & $11(1)$ & $3(1)$ \\
$\mathrm{C}(4)$ & $47(2)$ & $39(2)$ & $44(2)$ & $3(1)$ & $7(1)$ & $6(1)$ \\
$\mathrm{C}(5)$ & $76(3)$ & $40(2)$ & $68(2)$ & $0(2)$ & $20(2)$ & $1(2)$ \\
$\mathrm{C}(6)$ & $48(2)$ & $34(2)$ & $49(2)$ & $4(1)$ & $14(2)$ & $3(1)$ \\
$\mathrm{C}(7)$ & $37(2)$ & $41(2)$ & $53(2)$ & $5(1)$ & $8(1)$ & $-2(1)$ \\
$\mathrm{C}(8)$ & $42(2)$ & $46(2)$ & $47(2)$ & $8(1)$ & $6(1)$ & $2(1)$ \\
$\mathrm{C}(9)$ & $42(2)$ & $34(2)$ & $48(2)$ & $1(1)$ & $12(1)$ & $-1(1)$ \\
$\mathrm{C}(10)$ & $41(2)$ & $46(2)$ & $55(2)$ & $8(2)$ & $4(2)$ & $-5(1)$ \\
$\mathrm{C}(11)$ & $48(2)$ & $50(2)$ & $53(2)$ & $14(2)$ & $1(2)$ & $0(2)$
\end{tabular}




$\begin{array}{lllllll}\mathrm{C}(12) & 33(2) & 36(2) & 57(2) & 3(1) & 9(1) & 0(1) \\ \mathrm{C}(13) & 36(2) & 41(2) & 51(2) & 5(1) & 10(1) & -1(1) \\ \mathrm{C}(14) & 52(2) & 52(2) & 52(2) & 11(2) & 11(2) & -2(2) \\ \mathrm{C}(15) & 58(2) & 47(2) & 74(2) & 20(2) & 20(2) & -4(2) \\ \mathrm{C}(16) & 47(2) & 38(2) & 78(2) & 4(2) & 16(2) & -9(2) \\ \mathrm{C}(17) & 42(2) & 42(2) & 57(2) & 1(2) & 9(2) & -3(1) \\ \mathrm{C}(18) & 45(2) & 38(2) & 42(2) & 4(1) & 12(1) & -1(1) \\ \mathrm{C}(19) & 43(2) & 47(2) & 54(2) & 3(2) & 7(2) & 5(1) \\ \mathrm{C}(20) & 64(2) & 40(2) & 47(2) & 10(2) & 0(2) & 1(2) \\ \mathrm{C}(21) & 47(2) & 42(2) & 57(2) & 9(2) & 14(2) & 8(1) \\ \mathrm{C}(22) & 48(2) & 41(2) & 45(2) & 5(1) & 17(2) & -1(1) \\ \mathrm{C}(23) & 60(2) & 45(2) & 46(2) & 6(2) & -2(2) & -4(2) \\ \mathrm{C}(24) & 67(3) & 73(3) & 62(2) & 4(2) & 27(2) & 12(2) \\ \mathrm{C}(25) & 64(2) & 57(2) & 74(2) & -20(2) & 29(2) & -7(2) \\ \mathrm{C}(26) & 101(4) & 49(2) & 104(3) & 10(2) & 51(3) & 18(2) \\ \mathrm{C}(27) & 76(3) & 91(3) & 109(4) & 14(3) & 17(3) & 29(3) \\ \mathrm{C}(28) & 62(3) & 69(3) & 128(4) & -8(3) & 22(3) & 1(2) \\ \mathrm{C}(29) & 74(3) & 65(3) & 114(4) & 13(2) & 48(3) & 3(2) \\ \mathrm{C}(30) & 83(4) & 108(4) & 139(5) & 21(4) & 37(4) & -5(3) \\ \mathrm{C}(31) & 138(6) & 109(4) & 85(4) & 17(3) & -14(4) & -10(4) \\ \mathrm{C}(32) & 204(8) & 136(6) & 83(4) & 15(4) & 56(5) & 68(6) \\ \mathrm{C}(33) & 107(5) & 136(6) & 192(7) & 23(5) & 92(5) & 26(4) \\ \mathrm{C}(34) & 111(5) & 111(5) & 207(7) & -30(5) & 67(5) & -22(4) \\ \mathrm{C}(35) & 127(5) & 105(4) & 170(6) & -29(4) & 82(5) & -12(4) \\ & & & & & & \end{array}$

Table 4. Hydrogen coordinates ( x 10^4) and isotropic displacement parameters $\left(\mathrm{A}^{\wedge} 2\right.$ x 10^3).

\begin{tabular}{lcccc}
\hline & $\mathrm{x}$ & $\mathrm{y}$ & $\mathrm{z}$ & $\mathrm{U}(\mathrm{eq})$ \\
\hline $\mathrm{H}(1 \mathrm{~A})$ & 2259 & 4760 & 3797 & 86 \\
$\mathrm{H}(1 \mathrm{~B})$ & 2961 & 5648 & 4791 & 86 \\
$\mathrm{H}(1 \mathrm{C})$ & 3526 & 4456 & 4623 & 86 \\
$\mathrm{H}(3)$ & 4367 & 7083 & 4485 & 56
\end{tabular}




\begin{tabular}{|c|c|c|c|c|}
\hline $\mathrm{H}(5 \mathrm{~A})$ & 6568 & 8990 & 3986 & 92 \\
\hline $\mathrm{H}(5 \mathrm{~B})$ & 5064 & 8851 & 4310 & 92 \\
\hline $\mathrm{H}(5 \mathrm{C})$ & 5210 & 9134 & 3162 & 92 \\
\hline $\mathrm{H}(7)$ & 4570 & 7785 & 857 & 53 \\
\hline $\mathrm{H}(8)$ & 5264 & 8955 & -317 & 54 \\
\hline $\mathrm{H}(10)$ & 9191 & 9520 & 1437 & 58 \\
\hline $\mathrm{H}(11)$ & 8516 & 8297 & 2589 & 61 \\
\hline $\mathrm{H}(14)$ & 7604 & 10403 & -2851 & 62 \\
\hline $\mathrm{H}(15)$ & 8345 & 12293 & -2184 & 70 \\
\hline $\mathrm{H}(16)$ & 8850 & 12840 & -358 & 66 \\
\hline $\mathrm{H}(17)$ & 8438 & 11519 & 784 & 58 \\
\hline H(19) & 8543 & 7895 & -998 & 58 \\
\hline $\mathrm{H}(20)$ & 5335 & 8861 & -2972 & 62 \\
\hline $\mathrm{H}(21)$ & 2286 & 3994 & 1595 & 57 \\
\hline $\mathrm{H}(23)$ & 5537 & 3021 & 3541 & 63 \\
\hline $\mathrm{H}(24)$ & 6399 & 5475 & 324 & 79 \\
\hline $\mathrm{H}(25)$ & 5727 & 3771 & 982 & 79 \\
\hline $\mathrm{H}(26)$ & 7409 & 3045 & 2199 & 96 \\
\hline $\mathrm{H}(27)$ & 9688 & 3920 & 2654 & 109 \\
\hline $\mathrm{H}(28)$ & 10371 & 5547 & 1918 & 105 \\
\hline $\mathrm{H}(29)$ & 8747 & 6313 & 788 & 97 \\
\hline $\mathrm{H}(30)$ & 8028 & 7683 & -4174 & 131 \\
\hline $\mathrm{H}(31)$ & 8522 & 6729 & -5670 & 139 \\
\hline $\mathrm{H}(32)$ & 10829 & 7035 & -6056 & 161 \\
\hline $\mathrm{H}(33)$ & 12455 & 8411 & -4954 & 164 \\
\hline $\mathrm{H}(34)$ & 11893 & 9257 & -3429 & 173 \\
\hline $\mathrm{H}(35)$ & 9686 & 8923 & -3085 & 159 \\
\hline
\end{tabular}

